\title{
Improving the Mining Enterprise Productivity Based on Probabilistic Nature of the Solid Minerals Extraction and Transportation
}

\author{
Alexey Tyurin ${ }^{1, *}$, and Adrian Stoianov ${ }^{2}$ \\ ${ }^{1}$ T.F. Gorbachev Kuzbass State Technical University, Department of Automobile Transportation, \\ 650000, Kemerovo, Russian Federation \\ ${ }^{2}$ University of Petroșani, Faculty of Mining, 332006 Universității Str. 20 Petroșani, Romania
}

\begin{abstract}
Reducing costs in the extraction and transportation of solid minerals is an urgent task. To solve it, the article proposes the use of a systematic approach to consider the features of planning the operation of motor vehicles in conditions of coal mine functioning uncertainty. The account of the uneven operation of mining, transport units and complexes for processing and loading coal into railroad cars allows you to identify the shortcomings in the work of the entire enterprise and reduce resources use at the planned production level. On the example of the Kemerovo region LLC «Razrez Pervomaisky» in the article the model of the excavatorautomobile complex work planning is considered taking into account the dynamics of production and transport areas resources use. The use of random data makes it possible to flexibly redistribute vehicles along the routes of transportation and minimize the costs associated with the operation of dump trucks and excavators. The proposed methodology for planning the work of the excavator-automobile complex will allow to level the work and optimize the resources of all sections of the coal mine. The application of the proposed methods will free up unnecessary resources of the coal mine and redirect them to the needy facilities.
\end{abstract}

\section{Introduction}

The increase in the productivity of the mining enterprise is achieved, first of all, by considering the enterprise as a micrologistic system and analyzing the use efficiency of all resources involved in the extraction and promotion of solid minerals to the consumer.

First of all, it is necessary to assess the efficiency of the enterprise at the stages of mining and preparatory works (excavation, transportation, storage of rock mass) and mining (excavation, transportation, storage of minerals). In this regard, it is necessary to consider the work of the excavator-automobile complex as a stochastic subsystem with varying volumes of mining and transportation of rock mass and minerals.

\footnotetext{
* Corresponding author: alexturin07@,rambler.ru
} 
A lot of work has been devoted to the problem of planning the operation of motor vehicles in conditions of uncertainty. For example, it is possible to single out works that describe different situations of transport management in different production and logistic systems. So in [1-3] different dynamic and stochastic models of routing with casual demand for transport are described, leading to different solutions depending on the conditions of the problem.

In conditions of minerals extraction by the open method, the use of motor vehicles as part of the excavator-automobile complex is considered in [4-6]. In these studies, approaches are proposed to reduce downtime of the excavator-automobile complex, which most often arise due to improper planning and fixation of dump trucks behind excavators. The use of a closed cycle of excavator-automobile complex operation, in which the fleet is permanently assigned to a certain excavator, leads to an increase in idle dump times, a decrease in their productivity and an increase in the cost of the rock mass transportation. Since the work of subdivision units can be considered as a dynamic system [7-10], it becomes necessary to use an open cycle of excavator-car complex operation, in which a group of excavators serves a group of dump trucks in order to minimize downtime, increase productivity and reduce costs from excavator-automobile complex.

Thus, the system of planning dump trucks for a certain period of time (for example, a week) consists in the distribution of fleet for two types of trips: regular and special. Regular trips are held between fixed points and are planned for each week in advance. In conditions of an open method of mining, regular trips refer to a closed cycle of an excavatorautomobile complex operation. However, during the week plans may change due to the unevenness and randomness of the coal mine areas work [11-15]. Special trips occur irregularly, the time and points of transportation are not fixed in advance. Special trips can distract the fleet from regular lines.

Different types of vehicles differ in payload, trip time and costs on different routes. Trips are planned in conditions of incomplete information. The demand for special transports is unknown in advance. After a certain time, information is received about the uncertain parameters of the task's conditions. There is a need to reassign vehicles between routes. It is required to minimize the average expected costs for the entire planning period. The problem is posed as a two-stage stochastic problem. On the first stage, vehicles of each type are distributed between routes and the number of vehicles trips of each type for each line is determined. At the second stage, after the random parameters of the task conditions are established, the vehicles are reassigned from the route to the route. The fixed conditions (the conditions of the first stage) are limited from above for vehicles of each type by the total number of hours per week. Restrictions of the second stage can be divided into two groups. The limitations of the first group record the fact that for each type of vehicles the total number of hours transferred from this route to other lines does not exceed the number of hours originally assigned to this route. Restrictions of the second group, usual for twostage tasks of stochastic programming, are the balance relations for each route.

\section{Materials and methods}

Let us introduce the following notations: $x_{i j}$ - the number of trips during a week of vehicles of type $i$ initially assigned to route $j ; x_{i j k}$ - the number of trips of type $i$ vehicles taken off route $j$ and reassigned to route $k ; y_{j}^{+}-$unsatisfied applications (in tons of cargo) for transportation along route $j ; y \bar{j}$ — unloaded capacity of vehicles (in tons of 
cargo) on the $j$-th route; $a_{i j}$ - the number of hours required by a vehicle of type $i$ to overcome route $j$, if the vehicle was assigned to this route from the very beginning; $a_{i j k}$ - the number of hours required for a vehicle of type $i$, originally assigned to route $j$, in order to overcome route $k$. It is obvious, $a_{i j k} \geq a_{i j} ; b_{i j}$ - the number of tons of cargo transported in one trip by a vehicle of type $i$ along route $j ; a_{i}$ - the number of hours allowed for the trips by a vehicle of type $i ; d j-$ requests for shipment (in tons of cargo) along route $j ; c_{i j}$ - the cost of a vehicle trip of type $i$ along route $j$, provided that the vehicle has been assigned to this route from the very beginning; $c_{i j k}$ - the cost of a vehicle trip of type $i$ on route $k$, if it was taken off route $j$. Obviously, $c_{i j k} \geq c_{i j} ; q_{j}^{+}$ penalty for non-satisfaction of an application for the carriage of a ton of cargo along route $j$; $q \bar{j}$ - penalty for underload per one ton of the vehicle on route $j$.

Let us write the formal model of the problem in the notation introduced.

The conditions of the first stage, limiting for vehicles of each type the total number of operation hours along all routes, have the form

$$
\sum_{j} a_{i j} x_{i j} \leq a_{i}, \forall i
$$

To formalize the limitations of the second stage, it is necessary to take into account the following remark. The duration of a vehicle trip of type $i$, assigned to route $j$, is equal to $a_{i j}$ hours. If this vehicle is reassigned to route $k$, then crossing this route will take $a_{i j k}$ hours. Thus, this trip on route $k$ causes the cancellation $a_{i j k} / a_{i j}$ of trips along route $j$.

The conditions of the second stage of the first group, meaning that it is impossible to cancel more trips of type $i$ vehicles along route $j$ than originally planned for this route, are recorded in the form

$$
\sum_{k \neq j} \frac{a_{i j k}}{a_{i j}} x_{i j k} \leq x_{i j}, \forall i, j
$$

The conditions of the second group of the second stage have the form

$$
\sum_{i} b_{i j} x_{i j}+\sum_{i} \sum_{k \neq j} b_{i k} x_{i j k}-\sum_{i} \sum_{k \neq j}\left(b_{i j} \frac{a_{i j k}}{a_{i j}}\right) x_{i j k}+y_{j}^{+}-y_{j}=d_{j}, \forall j
$$

These are the balance conditions that determine the applications for transportation and their satisfaction.

The objective of the two-stage trip planning problem is expressed as follows:

$$
\sum_{i, j} c_{i j} x_{i j}+M\left\{\min _{x_{i j k}, y_{j}^{+}, y_{j}^{-}}\left[\sum_{i, j k \neq j}\left(c_{i j k}-c_{i j} \frac{a_{i j k}}{a_{i j}}\right) x_{i j k} \sum_{i}\left(q_{j}^{+} y_{j}^{+}-q \bar{j} y \bar{j}\right)\right]\right\}
$$


The task of trip planning is thus reduced to a two-stage model of stochastic programming, in which it is required to calculate non-negative parameters $x_{i j}, x_{i j k}, y_{j}^{+}, y_{j}$ minimizing the objective (4) under conditions (1) - (3). In addition, additional requirements for integers are imposed on variables $x_{i j}, x_{i j k}$.

\section{Results and discussion}

Let's consider the practical implementation of the model presented above on the example of the functioning of the excavator-automobile complex LLC «Razrez Pervomaisky».

The P\&H 2800 XPC, HITACHI EX5500, HITACHI EX3600, KOMATSU PC4000 excavators work on the coal mine with vehicles. The fleet consists of BelAZ-75137 and BelAZ-75309 models.

Transportation of rock mass is carried out from 4 different types of excavators to 3 unloading areas with different haulage distances. Figure 1 shows schematically the transport links between excavators and unloading areas.

Therefore, the transportation of rock mass is carried out along 5 routes by dump trucks of 2 types BelAZ-75137 and BelAZ-75309. The initial data are presented in Tables 1-2.

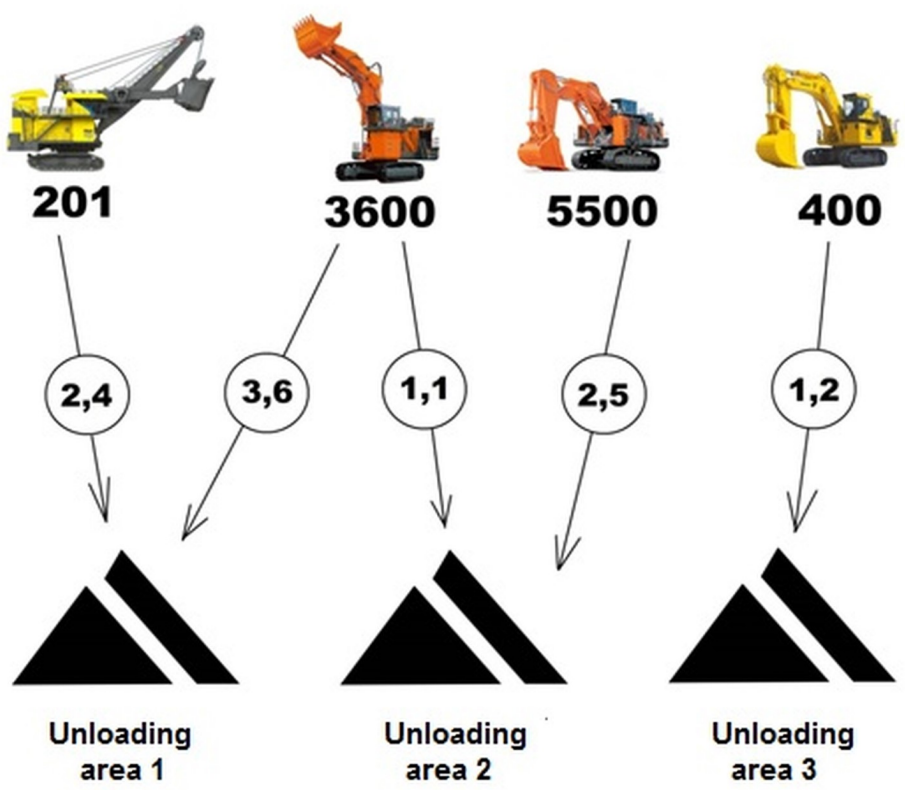

Fig. 1. Transport links between excavators and unloading areas.

Table 1. The trip time for 2 types of vehicles on 5 routes

\begin{tabular}{|c|c|c|c|c|c|}
\hline \multirow{2}{*}{$\begin{array}{c}\text { A type } \\
\text { the vehicle }\end{array}$} & \multicolumn{5}{|c|}{ Trip time (round trip). hours } \\
\cline { 2 - 6 } & Route 1 & Route 2 & Route 3 & Route 4 & Route 5 \\
\hline 1 & 0,274 & 0,284 & 0,389 & 0,19 & 0,203 \\
\hline
\end{tabular}


In addition, the demand for transport can be deterministic or random with average values along the routes in the amount of $16,000,12,000,2,700,6300$ and 5,900 tons. In addition, the time to switch between any routes is 1 hour for both types of vehicles. Increasing costs in connection with the reassignment of vehicles will be 1500 and 2600 rubles for two types of vehicles respectively. It is also assumed that the total working time for each type of vehicle per week is 40 hours.

The optimal solution to the problem (variant 1) is presented in Table 3. Analysis of the data obtained shows that initially 77 trips of a type 1 vehicle were planned for route 1,60 trips on route 2 and 5 trips on route 3 . For the type 2 vehicle, originally 46 trips were planned for route 1, 4 trips for route 3,33 trips for route 4 and 32 trips for route 5 .

Table 2. Initial data on routes

\begin{tabular}{|c|c|c|c|c|}
\hline \multicolumn{5}{|c|}{ Carrying capacity (in tons) } \\
\hline Route 1 & Route 2 & Route 3 & Route 4 & Route 5 \\
\hline 130 & 130 & 130 & 130 & 130 \\
\hline 220 & 220 & 220 & 220 & 220 \\
\hline \multicolumn{5}{|c|}{ Cost per trip, RUB } \\
\hline 1372 & 1430 & 2059 & 629 & 686 \\
\hline 2640 & 2750 & 3960 & 1210 & 1320 \\
\hline The penalty (rubles per ton) with excessive demand \\
\hline 792 & 825 & 1188 & 363 & 396 \\
\hline The penalty (rubles per ton) with excessive supply \\
\hline 274 & 286 & 411 & 125 & 137 \\
\hline
\end{tabular}

Table 3. Optimum values for 1 and 2 variants

\begin{tabular}{|c|c|c|c|c|c|c|c|c|c|c|}
\hline \multicolumn{10}{|c|}{ Variant 1 } & \multicolumn{1}{|c|}{ Variant 2 } \\
\hline \multicolumn{10}{|c|}{$x_{i j k}$ for the first type of vehicle } \\
\hline 77 & 60 & 5 & 0 & 0 & 156 & 60 & 6 & 40 & 50 \\
\hline 46 & 0 & 4 & 33 & 32 & 0 & 0 & 5 & 6 & 0 \\
\hline \multicolumn{10}{|c|}{} \\
\hline 0 & 17 & 0 & 0 & 0 & 0 & 33 & 0 & 0 & 0 \\
\hline 0 & 0 & 8 & 0 & 0 & 0 & 0 & 7 & 0 & 0 \\
\hline 0 & 0 & 0 & 0 & 0 & 0 & 0 & 0 & 2 & 3 \\
\hline 0 & 0 & 0 & 0 & 0 & 0 & 7 & 0 & 0 & 0 \\
\hline 0 & 0 & 0 & 0 & 0 & 0 & 2 & 8 & 0 & 0 \\
\hline & & & $x_{i j k}$ for the second type of vehicle & & \\
\hline 0 & 9 & 0 & 0 & 0 & 0 & 0 & 0 & 0 & 0 \\
\hline 0 & 0 & 0 & 0 & 0 & 0 & 0 & 0 & 0 & 0 \\
\hline 0 & 0 & 0 & 0 & 0 & 0 & 0 & 0 & 0 & 0 \\
\hline
\end{tabular}




\begin{tabular}{|l|l|l|l|l|l|l|l|l|l|}
\hline 0 & 5 & 0 & 0 & 0 & 0 & 2 & 0 & 0 & 0 \\
\hline 0 & 2 & 2 & 0 & 0 & 0 & 0 & 0 & 0 & 0 \\
\hline \multicolumn{10}{|c|}{$y_{j}^{+}$} \\
\hline 0 & 0 & 0 & 0 & 0 & 0 & 0 & 0 & 0 & 0 \\
\hline \multicolumn{10}{|c|}{$y \bar{j}$} \\
\hline 0 & 0 & 0 & 0 & 0 & 0 & 0 & 0 & 0 & 0 \\
\hline
\end{tabular}

After specification of demand and reassignment of vehicles the following situation was presented: vehicles of 1 type are removed from route 1 and are reassigned in the amount of 17 trips to route 2 , from route 2 are reassigned in the number of 8 trips to route 3 . For vehicles of the 2 nd type, the redistribution leads to a destination for route 2, which were removed from routes 1,4 and 5 in the number of 9,5 and 2 trips respectively. If the total working time for each type of vehicle per week is increased to 80 hours (2 drivers are assigned to 1 vehicle), then the optimal solution of the problem (variant 2) will be different (see Table 3).

An analysis of the data shows that 156 trips of type 1 vehicle were originally planned for route 1, 60 trips for route 2, 6 trips for route 3, 40 trips for route 4 and 50 trips for route 5. The second type of vehicle is planned for routes 3 and 4 in the number of 5 and 6 trips respectively. After specifying the demand and reassignment of vehicles, we get: 1 type vehicles are removed from routes 1,4 and 5 in the amount of 33.7 and 2 trips respectively and are reassigned to route 2 . Additionally, vehicles of type 1 are removed from route 2 and reassigned to route 3 in the number 7 In conclusion, vehicles of type 1 are taken off route 3 and reassigned to routes 4 and 5 in the number of 2 and 3 trips respectively. For type 2 vehicles, redistribution will result in de-route 4 and reassignment in the number of 2 trips to route 2. Zeros under the variables $y_{j}^{+}$and $y_{j}$ show that the requests for transportation are fully satisfied and the loading of cars for the flight is in compliance with the regulations.

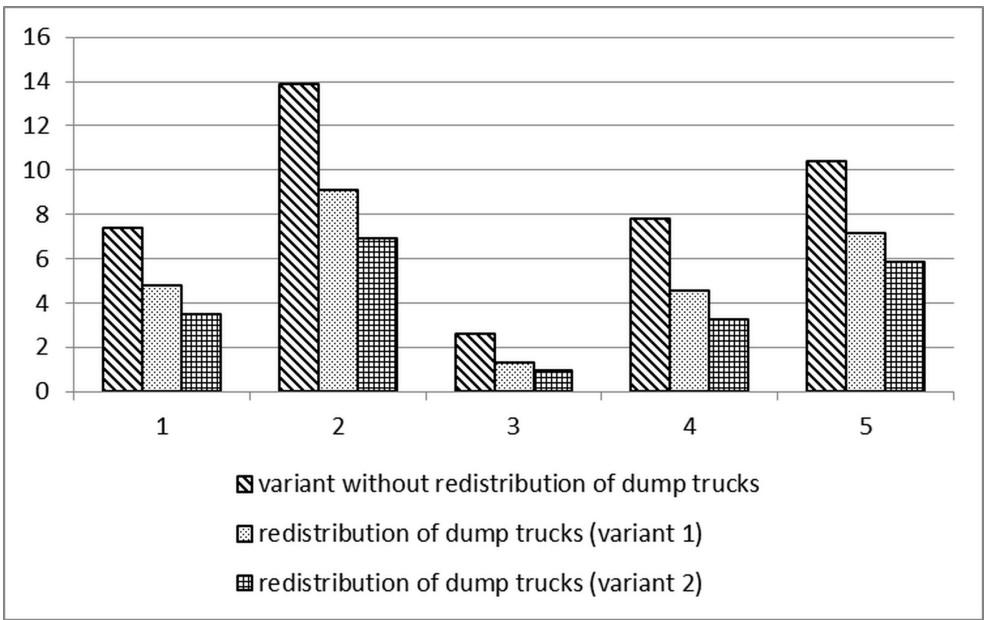

Fig. 2. Reduced productivity (\%) for different variants of dump trucks operation on different routes.

In the initial version of the excavator-vehicle complex (without redistribution of dump trucks), downtime of vehicles on different routes ranges from $2 \%$ to $14 \%$ of the total operating time. Consequently, there is a loss of productivity for the transportation of rock 
mass to the dump. In the case of redistribution of dump trucks, the share of their downtime is reduced from $1.5 \%$ to $9 \%$ (variant 1 ) and from $1 \%$ to $7 \%$ (variant 2) (figure 2). Taking into account the different volumes of traffic on the routes, the weighted average loss of productivity without redistribution of vehicles will be $9.4 \%$, and for 1 and 2 variants of redistribution, respectively, $6.1 \%$ and $4.5 \%$. Consequently, when using the redistribution of rolling stock along transport routes, there is an increase in productivity by $3.3-4.9 \%$, depending on the redistribution variant used.

\section{Conclusion}

The examples discussed above show the importance of accounting for temporary restrictions on the performance of trips. The use of a mechanism for the redistribution of dump trucks along transportation routes improves the productivity of the mining enterprise. The cost indicators of the task regulate the priority of trips and the degree of loading of the fleet within a week. Therefore, a detailed accounting of all input parameters and conditions of customer service will allow to develop a plan for the work of vehicles for any time interval, taking into account the dynamism of demand, the quantitative composition of vehicles with minimal costs of transport services. The proposed methodology for planning the work of the excavator-automobile complex will allow to level the work and optimize the resources of all sections of the coal mine. The application of the proposed methods will free up unnecessary resources of the coal mine and redirect them to the needy facilities.

\section{References}

1. Bent R., Hentenryck P., Oper. Res., 52:6, 977 (2004)

2. Bertsimas D., Ryzin G. V., Oper. Res., 39:4. 601 (1991)

3. Bertsimas D., Oper. Res., 40:3, 574 (1992)

4. Bertsimas D., Ryzin G. V., Oper. Res., 41:1, 60 (1993)

5. Carter R.A., Eng. Min. J., 32, 28 (2012)

6. M. Munirathinam, J.C. Yingling, Int J Surf Min Reclamat Environ, 8, 1 ( 1994)

7. S. Alarie, M. Gamache, Int J Surf Min Reclamat Environ, 16, 59 (2002)

8. C.H. Ta, J.V. Kresta, J.F. Forbes, H.J. Marquez, Int J Surf Min Reclamat Environ, 19, $162(2005)$

9. A. Krause, C. Musingwini, J. S. Afr. Inst. Min. Metall., 107, 469 (2007)

10. C.N. Burt, L. Caccetta, Int J Surf Min Reclamat Environ, 21, 263 (2007)

11. Krzyzanowska J., J. S. Afr. Inst. Min. Metall., 107, 215 (2007)

12. Mkhatshwa S.V., J. S. Afr. Inst. Min. Metall., 109, 223 (2009)

13. K.P. Chatterjee, J.D. Brake, CIM Bull., 74, 103 (1981)

14. Y.C. Kim, M.A. Ibarra, Bulk Solids Handling, 1, 137 (1981)

15. J.H. Tu, V.J. Hucka, CIM Bull., 78, 53 (1985) 